\title{
Temporal Frequency Spread of Plane Wave Propagation through Moderate to Strong Turbulence
}

\author{
Bing Guan, Jaeho Choi
}

\begin{abstract}
In this study, we derive new expressions for the atmospheric-brought on frequency unfold of plane waves propagating thru slight to strong turbulence in a horizontal direction based on the modified anisotropic non-Kolmogorov electricity spectrum as antagonistic to conventional Kolmogorov electricity spectrum. The energy regulation price varies from three to 4 instead of the traditional Kolmogorov power law price; the general amplitude price differs from the conventional Kolmogorov regular cost 0.033. these new expressions are based on slight to robust fluctuation vicinity and anisotropic non-Kolmogorov turbulence. The simulation results show that temporal frequency

unfold will decrease even as the anisotropic component $\varsigma^{2-\alpha}$ is increasing.
\end{abstract}

Index Terms: moderate to strong turbulence, frequency spread, anisotropic turbulence.

\section{INTRODUCTION}

The everyday Kolmogorov model has been comprehensively achieved on the test and hypothetical examinations roughly laser pillar spread through abnormal media for a protracted haul [1]. These days we have discovered that the normal Kolmogorov variation is not usually the high-quality fierce in the surroundings, there are greater non-Kolmogorov disturbance designs have been utilized [2-9]. Through the usage of the variety that does exclude the inward and external scales, the Kolmogorov variety now and again does no longer portray pleasantly the environmental choppiness facts [2]. Based clearly at the all-inclusive Rytov estimation idea, the changed anisotropic choppiness refractive-listing vacillations unearthly model is determined depending on the drawn out Rytov wager thought for the hypothetical examinations of optical aircraft and circular waves engendering through moderate-to-solid anisotropic non-Kolmogorov disturbance [3, 4]. The brand new articulations of barometrical expedited recurrence unfurl for aircraft wave and spherical wave in powerless anisotropic non-Kolmogorov choppiness were decided [5]. For contemplating the debasing aftereffects of environmental disturbance on an imaging device that may be defined by techniques for the air tweak switch work (MTF), a pristine articulation for the wave form trademark (WSF) of Gaussian-bar dependent on the susceptible variance idea were decided [6]. The systematic articulations for the lengthy haul fundamental air accelerated recurrence spread of optical waves engendering via clean air disturbance have been included [7]. The idea of anisotropy at amazing environmental choppiness scales has been covered and a electricity range and its related shape work with inward and external scale results and anisotropy had been established [8]. With the decided SI and the gamma-gamma appropriation, blur attainable effects and propose blur time for FSO systems are inferred [9].

On this paper the worldly recurrence unfurl of the air ship wave that proliferates inside the moderate to effective disturbance is tested. On this route, the pristine articulation of the worldly recurrence unfurl of the flying device wave proliferating in the moderate to solid anisotropic non-Kolmogorov choppiness is determined. At that factor, we take a look at the affect of the power guideline type and anisotropic perspective and the space fee $\mathrm{L}$ on the brief recurrence unfurl.

\section{ANISOTROPIC NON-KOLMOGOROV energy SPECTRUM}

At the factor whilst the laser bar engendering along a fair heading and anisotropic choppiness, the best variety is given with the manual of [2] as pursues: $\Phi_{n}(\kappa, \alpha, \varsigma)=A(\alpha) \cdot{C_{n}^{2}}^{2} \varsigma^{2} \cdot\left[\kappa_{z}^{2}+\varsigma^{2} \cdot\left(\kappa_{x}^{2}+\kappa_{y}^{2}\right)\right]^{-\frac{\alpha}{2}}$, $\left(1 / L_{0}<\kappa<1 / I_{0}, 3<\alpha<4\right)$,

$$
A(\alpha)=\frac{1}{4 \pi^{2}} \cdot \Gamma(\alpha-1) \cdot \cos \left[\frac{\alpha \pi}{2}\right],(3<\alpha<4),
$$

where $\alpha$ is the power law exponent value; $\zeta$ is the anisotropic factor value; $\Gamma(\cdot)$ is the gamma function that can be found in [10]. ${ }^{C_{n}^{2}}$ Is

with unit $\mathrm{m} 3-\alpha$, which is defined within the famous Hufnagel-Valley (HV) equation [11]; L0 is the outer scale size of the eddy; 10 is the internal scale length of the eddy. Is the wave range of the eddy length of turbulence; $\kappa_{x}, \kappa_{y}, \kappa_{z}$ are the components of $\kappa$ in $\mathrm{x}, \mathrm{y}$ and $\mathrm{z}$ direction.
Revised Version Manuscript Received on August 19, 2019.

Bing Guan, Dept. of Information Engineering, Eastern Liaoning University, Dandong 18003, China.

Jaeho Choi, Dept. of Electronic Engineering, CAIIT, Chonbuk National University, Chonju, 561-756, Rep. of Korea. 
When $\kappa_{Z}$ can be ignored for the horizontal path, then, rewritten as follows:

$$
\left.\Phi_{n}(\kappa, \alpha, \varsigma)=A(\alpha) \cdot \hat{C}_{n}^{2} \cdot \varsigma^{2-\alpha} \cdot \kappa^{-\alpha}, \kappa=\sqrt{\kappa_{x}^{2}+\kappa_{y}^{2}},(3<\alpha<4), 3\right)
$$

In the slight to strong anisotropic non-Kolmogorov choppiness, the modified anisotropic disturbance as pursues:

$$
\begin{aligned}
& \Phi_{n 1}(\kappa, \alpha, \varsigma)=\Phi_{n}(\kappa, \alpha, \varsigma) \cdot G(\kappa, \alpha, \varsigma),(3<\alpha<4), \\
& G(\kappa, \alpha, \varsigma)=G_{x}(\kappa, \alpha, \varsigma)+G_{y}(\kappa, \alpha, \varsigma), \\
& G_{x}(\kappa, \alpha, \varsigma)=\exp \left[-\frac{\kappa^{2}}{\kappa_{x}^{2}(\alpha, \varsigma)}\right], \\
& G_{y}(\kappa, \alpha, \varsigma)=\frac{\kappa^{\alpha}}{\left[\kappa^{2}+\kappa_{y}^{2}(\alpha, \varsigma)\right]^{\alpha / 2}}, \\
& \text { where } G_{x}(\kappa, \alpha, \varsigma) \text { and } G_{X}(\kappa, \alpha, \varsigma), G_{y}(\kappa, \alpha, \varsigma), \\
& \kappa_{x}(\alpha, \varsigma) \kappa_{y}(\alpha, \varsigma) \text {; these terms are found in [4]. }
\end{aligned}
$$

\section{TEMPORAL FREQUENCY spread OF plane WAVE}

An atmospheric-triggered frequency spread in optic wave feature has been stated in [7],

$$
\sigma_{f}^{2}=-\left.\frac{B_{s}^{\prime \prime}(\tau)}{4 \pi^{2}}\right|_{\tau=0},
$$

where $\sigma_{f}^{2}$ is the frequency variance and $B_{s}(\tau)$ is the temporal phase covariance, the plane wave temporal phase covariance given in [1] is modified as

where $\mathrm{J}_{0}($.$) denotes the Bessel function defined in$ [10].After the second derivate of ${ }^{B_{S}}(\tau)$, Eq. (8) can be de defined as follows:

$$
\begin{aligned}
& \sigma_{f, p 1}^{2}=0.25 k^{2} \cdot L \cdot V^{2} \cdot \int_{0}^{1} \int_{0}^{\infty} \kappa^{3} \cdot \Phi_{n 1}(\kappa, \alpha, \varsigma) \\
& \times\left[1+\cos \left(\frac{L \kappa^{2} \varepsilon}{k}\right)\right] d \kappa d \varepsilon,
\end{aligned}
$$

Utilizing the power range given in Eq. (4) we can separate the articulation into an aggregate of two sections, i.e., I1 and I2. The initial segment is as per the following:

$$
\sigma_{f, p l}^{2}\left(I_{1}\right)=0.25 k^{2} \cdot L \cdot V^{2} \cdot \int_{0}^{\infty} \kappa^{3} \cdot \Phi_{n 1}(\kappa, \alpha, \varsigma) d \kappa
$$

Using the gamma function given in [10]

$$
\Gamma(x)=\int_{0}^{\infty} k^{x-1} \cdot e^{-k} d k(k>0, x>0),
$$

One can obtain the first result I1 as follows: $\kappa=\sqrt{\kappa_{z}^{2}+\varsigma^{2} \cdot\left(\kappa_{x}^{2}+\kappa_{y}^{2}\right)}$, and also Eq. (1) can be refractive-record vacillations phantom model is given by [3]

$$
\begin{aligned}
& B_{s, p l}(V, \tau)=2 \pi^{2} \cdot k^{2} \cdot L \cdot \int_{0}^{1} \int_{0}^{\infty} \kappa \cdot \Phi_{n 1}(\kappa, \alpha, \varsigma) \cdot J_{0}(\kappa V \tau) \\
& \times\left[1+\cos \left(\frac{L \kappa^{2} \varepsilon}{k}\right)\right] d \kappa d \varepsilon,
\end{aligned}
$$

$$
\begin{aligned}
& \sigma_{f, p l}^{2}\left(I_{1}\right)=R(\alpha) \cdot \sigma_{R}^{2} \cdot V^{2} \cdot \varsigma^{2-\alpha} \cdot\left[\kappa_{x}^{4-\alpha}(\alpha, \varsigma) \cdot \Gamma\left(2-\frac{\alpha}{2}\right)\right. \\
& \left.+\kappa_{y}^{4-\alpha}(\alpha, \varsigma) \cdot \frac{\Gamma\left(\frac{\alpha}{2}-2\right)}{\Gamma\left(\frac{\alpha}{2}\right)}\right],
\end{aligned}
$$

and $R(\alpha)$ and ${ }^{2}{ }_{R}^{2}(\alpha)$ are defined as follows:

$$
\begin{aligned}
& R(\alpha)=-\frac{\alpha}{64 \pi^{2}}\left[\Gamma\left(1-\frac{\alpha}{2}\right) \cdot \sin \left(\frac{\pi \alpha}{4}\right)\right]^{-1} \cdot k^{\frac{\alpha}{2}-1} \cdot L^{1-\frac{\alpha}{2}}, \\
& \sigma_{R}^{2}(\alpha)=-8 \pi^{2} \cdot A(\alpha) \cdot \frac{1}{\alpha} \cdot \Gamma\left(1-\frac{\alpha}{2}\right) \cdot \sin \left(\frac{\pi \alpha}{4}\right) \cdot{C_{n}^{2}}_{n} \cdot k^{3-\frac{\alpha}{2}} \cdot L^{\frac{\alpha}{2}},
\end{aligned}
$$

where ${ }_{R}^{2}(\alpha)$ is the non-Kolmogorov Rytov variance. In addition, the second part $\mathrm{I} 2$ is defined as follows:

$$
\begin{aligned}
& \sigma_{f, p l}^{2}\left(I_{2}\right)=0.25 k^{2} \cdot L \cdot V^{2} \cdot \sum_{n=0}^{\infty} \frac{\left(-L^{2} / k^{2}\right)^{n}}{(2 n) !} \\
& \times \int_{0}^{1} \varepsilon^{2 n} d \varepsilon \cdot \int_{0}^{\infty} \kappa^{4 n+3} \cdot \Phi_{n}(\kappa, \alpha, \varsigma) d \kappa,
\end{aligned}
$$

we can get the result of $\mathrm{I} 2$ as follows:

$$
\begin{aligned}
& \sigma_{f, p l}^{2}\left(I_{2}\right)=R(\alpha) \cdot \sigma_{R}^{2} \cdot V^{2} \cdot \varsigma^{2-\alpha} \cdot\left[\kappa_{x}^{4-\alpha}(\alpha, \varsigma) \cdot \Gamma\left(2-\frac{\alpha}{2}\right) \cdot J(\alpha, \varsigma)\right. \\
& \left.+\kappa_{y}^{4-\alpha}(\alpha, \varsigma) \cdot \frac{\Gamma\left(\frac{\alpha}{2}-2\right)}{\Gamma\left(\frac{\alpha}{2}\right)} \cdot K(\alpha, \varsigma)\right], \\
& J(\alpha, \varsigma)={ }_{2} F_{1}\left(1-\frac{\alpha}{4}, \frac{3}{2}-\frac{\alpha}{4} ; \frac{3}{2} ;-\frac{L^{2}}{k^{2}} \kappa_{x}^{4}(\alpha, \varsigma)\right), \\
& K(\alpha, \varsigma)={ }_{1} F_{2}\left(1 ; 2-\frac{\alpha}{4}, \frac{3}{2}-\frac{\alpha}{4} ;-\frac{L^{2}}{4 k^{2}} \kappa_{y}^{4}(\alpha, \varsigma)\right),
\end{aligned}
$$

where ${ }_{1} F_{2}$ and ${ }_{2} F_{1}$ are hypergeometric capacities found in [10]. At last, we can characterize the total articulation for the transient recurrence spread of the plane wave by taking the square foundation of the entirety of $\sigma_{f, p l}^{2}\left(I_{1}\right)$ and $\sigma_{f, p l}^{2}\left(I_{2}\right)$

\section{RESULTS AND DISCUSSION}

We investigate the barometrical initiated recurrence unfold of the aircraft wave engendering thru moderate to solid anisotropic non-Kolmogorov disturbance making use of the outcomes inferred inside the above location. We applied MATLAB to do the undertaking work. This plane wave is concept to engender with a wavelength .one of a kind parameters are $\mathrm{V}=10 \mathrm{~km} \mathrm{~h}-1$, and $10=10 \mathrm{~mm}$; the period of the manner length $\mathrm{L}$ is $1000 \mathrm{~m}$. The worldly recurrence spread influences initiated through the power law kind esteem and the anisotropic issue are seemed in Fig. 1. The environment disturbance is isotropic when the estimation of the anisotropic factor is 1 . The fleeting recurrence of aircraft wave inside the mild to strong anisotropic non-Kolmogorov

Published By: 
choppiness is expanding whilst shifts from three to a few.1; at that factor diminishing from 3.1 to a few.nine; at lengthy final, expanding from three. 9 to 4 . This parent additionally demonstrates the anisotropic factor s impact on the fleeting recurrence spread of the plane wave for its estimations of one, five, 7, and 10, in my opinion. This plot indicates that the anisotropic issue can lessen the transient recurrence spread whilst is expanding. at the factor when the 's really worth equivalents 1, the choppiness is isotropic. For distinctive characteristics, the choppiness finishes up anisotropic. From the aftereffects of the duplicate we are able to discover that the fleeting recurrence spread is converting all the extra absolutely by means of the extraordinary strength kind esteem when the choppiness is isotropic. on the point when the anisotropic component 's well worth increments from 5 to 10 , the disturbance will wind up anisotropic, and the worldly recurrence unfold do not alternate honestly whilst the energy instance really worth is evolving. Likewise, inside the anisotropic choppiness, the fleeting recurrence spread is most massive whilst 's esteems are around three.1. The modifications inside the qualities are sensitive to the temperature sorts which might be obvious among the layers of the Earth environment. The Earth's air is isolated into 4 vital layers. the bottom layer is the troposphere achieving out up to eleven $\mathrm{km}$ and it includes generally $75 \%$ of the Earth's air mass. Stratosphere is the layer over the tropopause, and it stretches out from $20 \mathrm{~km}$ as much as $48 \mathrm{~km}$ in peak. Mesosphere is the layer stretching out from the stratopause to about eighty $\mathrm{km}$ excessive. At lengthy last, thermosphere is the layer attaining out from the mesopause to around $600 \mathrm{~km}$ in top [1]. as the elevation increment alongside the layers of the Earth weather, the electricity law kind well worth changes from 10/3 to a few.nine. From the reenactment effects, you can find that the disturbance impact receives more grounded within the troposphere layer than within the stratosphere layer.

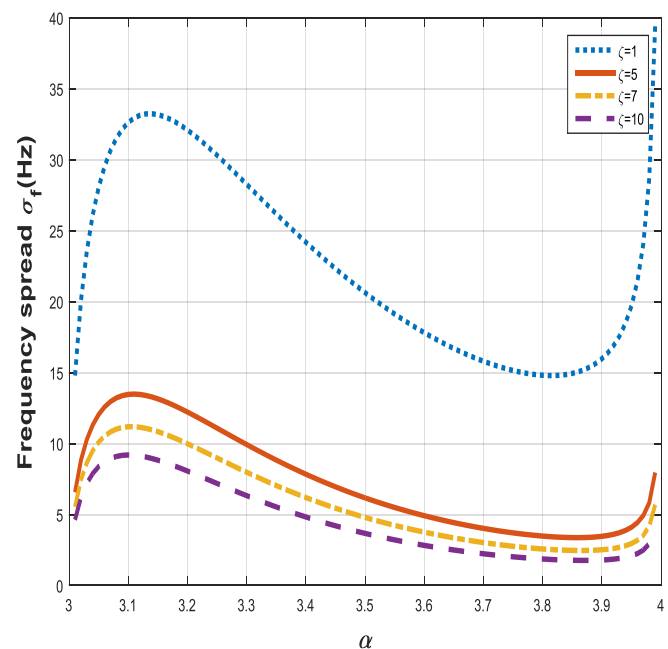

Fig.1. Plane wave frequency spread with respect to power law exponent and anisotropic factor

As shown in Fig.2, one can observe the frequency spread effects by the anisotropic factor $\mathcal{S}$ and the distance of propagation. The value of the power law exponent $\alpha$ is $11 / 3$ in this figure. When $\alpha$ varies between 3 and 4 , from Eq. (3), one can see that the exponent of the anisotropic factor $\varsigma^{2-\alpha}$ is always negative. In other words, the values of the anisotropic factor $\varsigma^{2-\alpha}$ decrease when $\alpha$ increases. The frequency spread is more obvious when the turbulence is isotropic. When the power exponent value $\alpha$ is $11 / 3$, the temporal frequency spread of plane wave is increasing when the propagation distance increases from 100 meters to 6000 meters. One can capture the effects of two parameters, $\alpha$ and $\varsigma^{2-\alpha}$

from the graphs shown in Fig. 2. As $\alpha$ increases, the value of the anisotropic factor $\varsigma^{2-\alpha}$ decrease and it reduces the temporal frequency spread.

On the other hand, as shown in Fig.3, one can observe the frequency spread effects by the power law exponent value $\alpha$ and the distance of propagation. When the anisotropic value $\checkmark$ is 1 , the temporal frequency spread of plane wave is increasing when the distance increases from 100 meters to 6000 meters while the temporal frequency decreases as the power exponent value $\alpha$ varies from 10/3 to 3.9. More specifically, from Eq. (14), one can expect the effect of the propagation distance. The equation indicates that the turbulence strength increases when the distance increases. In turn, the frequency spread becomes bigger when the turbulence strength increases. It means that the turbulence effect is more apparent when the type of turbulence is isotropic. Also, as far as the temperature variations along the layers of the Earth atmosphere, one can get the similar results for the graphs in Fig. 2; the turbulence effect gets stronger in the troposphere layer than in the stratosphere layer.

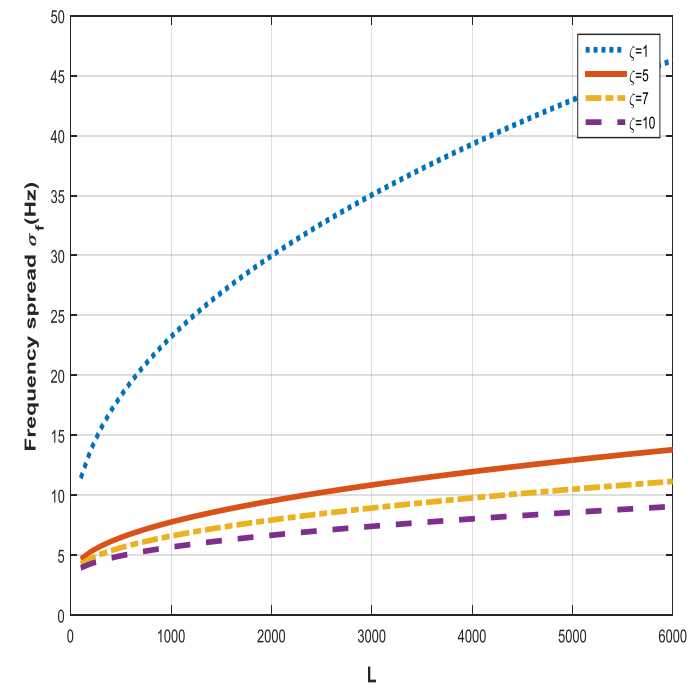

Fig.2. Plane wave frequency spread with respect to propagation distance and anisotropic factor 


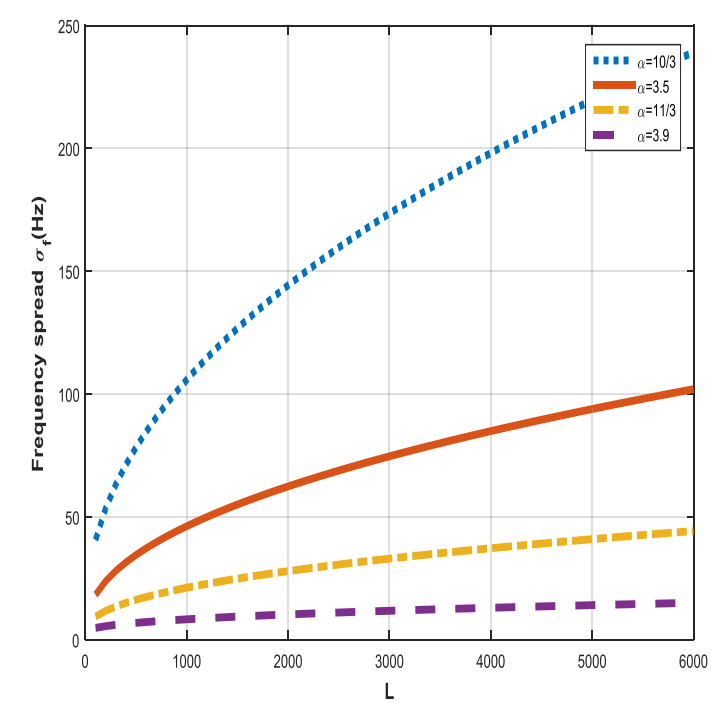

Fig.3. Plane wave frequency spread with respect to propagation distance and power law exponent

\section{CONCLUSIONS}

In this paper we have presented the influence of the atmospheric-induced frequency spread of plane wave in moderate to strong anisotropic non-Kolmogorov turbulence with respect to power law exponent, anisotropic factor, and propagation distance. We have used modified anisotropic non-Kolmogorov power spectrum on moderate to strong turbulence and derived new expressions of the temporal frequency spread of plane wave. The simulation results have shown us that the temporal frequency spread decreases as the anisotropic factor increases; also, the temporal frequency is well affected by the power exponent. In addition, as our guess, the temporal frequency spread increases when the propagation distance increases.

All of the derivations presented in this paper are based on the case of horizontal path's propagation. When the path is horizontal, $C_{n}^{2}$ can be a constant, but when the path becomes slant or vertical, $C_{n}^{2}$ will not be constant anymore as the altitude of the optical wave gets higher. In future study, the temporal frequency spread of optical wave propagating through the slant or vertical path will be analyzed.

\section{ACKNOWLEDGMENT}

This work has been supported partly by BK21+ and Chonbuk National University of Korea 2018.

\section{REFERENCES}

1. Andrews L C and Phillips R L, Laser Beam Propagationthrough Random Media 2nd ed. Bellingham, WA: SPIE2005

2. I. Toselli, B. Agrawal, S. Restaino, Light propagation through anisotropic turbulence, J. Opt. Soc. Am. A (2011) 28 (3) 483-488.

3. Cui L, Xue B, Zhou F, Modified anisotropic turbulence refractive-index fluctuations spectral model and its application in moderate-to-strong anisotropic turbulence, J Opt Soc Am A Opt Image Sci Vis(2016) 33(4):483-491.

4. Cui L, Atmosphere turbulence MTF models in moderate-to-strong anisotropic turbulence,Optik 130
(2017) 68-75.

5. Kotiang S, Choi J, Temporal frequency spread of optical wave propagation through anisotropic non-Kolmogorov turbulence, J. Opt. 17 (2015) 125606.

6. Kotiang S, Choi J, Wave structure function and long-exposure MTF for laser beam propagation through non-Kolmogorov turbulence, Optics \& Laser Technology 74 (2015) 87-92.

7. Masino A J and Young C Y, Atmospheric-inducedfrequency spread in optical waves J. Mod. Opt. 53(2006) 1879-99.

8. Toselli I, Introducing the concept of anisotropy at different scales for modeling optical turbulence. J Opt Soc Am A Opt Image Sci Vis (2014) 31(8)1868-1875.

9. Yi X, Liu Z, Yue P, Optical scintillations and fade statistics for FSO communications through moderate-to-strong non-Kolmogorov turbulence. Optics \& Laser Technology (2013) 47(47):199-207.

10. Andrews L C, Special Functions of Mathematics for Engineers, 2nd ed. McGraw-Hill, 1997.

11. Hufnagel R E, Variations of atmospheric turbulence. Proc.of Topical Meeting on Optical Propagation through the Turbulence, 1974. 